\title{
Data Management Tracking Document
}

National Cancer Institute

\section{Source}

National Cancer Institute. Data Management Tracking Document. NCI Thesaurus. Code C115684.

Records pertaining to any activities logged that relate to data management during the course of a clinical trial. 